\title{
Identification of new drug candidates against Borrelia burgdorferi using high-throughput screening
}

\author{
This article was published in the following Dove Press journal: \\ Drug Design, Development and Therapy \\ I April 2016 \\ Number of times this article has been viewed
}

\author{
Venkata Raveendra \\ Pothineni' \\ Dhananjay Wagh' \\ Mustafeez Mujtaba Babar' \\ Mohammed Inayathullah' \\ David Solow-Cordero ${ }^{2}$ \\ Kwang-Min Kim' \\ Aneesh V Samineni' \\ Mansi B Parekh' \\ Lobat Tayebi ${ }^{3}$ \\ Jayakumar Rajadas' \\ 'Biomaterials and Advanced Drug \\ Delivery Laboratory, Stanford \\ Cardiovascular Pharmacology \\ Division, Cardiovascular Institute, \\ Stanford University School of \\ Medicine, Palo Alto, ${ }^{2}$ Chemical \& \\ Systems Biology, Stanford University \\ School of Medicine, Stanford, CA, \\ ${ }^{3}$ Department of Developmental \\ Sciences, Marquette University School \\ of Dentistry, Milwaukee, WI, USA
}

Correspondence: Jayakumar Rajadas

Biomaterials and Advanced Drug Delivery Laboratory, Stanford Cardiovascular Pharmacology Division, Cardiovascular Institute, Stanford University School of Medicine, 1050 Arastradero Road, Building A, Room AI 48, Palo Alto,

CA 94304, USA

$\mathrm{Tel}+\mathrm{I} 6507246806$

Fax +I 6507244694

Email jayraja@stanford.edu

\begin{abstract}
Lyme disease is the most common zoonotic bacterial disease in North America. It is estimated that $>300,000$ cases per annum are reported in USA alone. A total of $10 \%-20 \%$ of patients who have been treated with antibiotic therapy report the recrudescence of symptoms, such as muscle and joint pain, psychosocial and cognitive difficulties, and generalized fatigue. This condition is referred to as posttreatment Lyme disease syndrome. While there is no evidence for the presence of viable infectious organisms in individuals with posttreatment Lyme disease syndrome, some researchers found surviving Borrelia burgdorferi population in rodents and primates even after antibiotic treatment. Although such observations need more ratification, there is unmet need for developing the therapeutic agents that focus on removing the persisting bacterial form of $B$. burgdorferi in rodent and nonhuman primates. For this purpose, high-throughput screening was done using BacTiter-Glo assay for four compound libraries to identify candidates that stop the growth of B. burgdorferi in vitro. The four chemical libraries containing 4,366 compounds (80\% Food and Drug Administration [FDA] approved) that were screened are Library of Pharmacologically Active Compounds (LOPAC1280), the National Institutes of Health Clinical Collection, the Microsource Spectrum, and the Biomol FDA. We subsequently identified 150 unique compounds, which inhibited $>90 \%$ of B. burgdorferi growth at a concentration of $<25 \mu \mathrm{M}$. These 150 unique compounds comprise many safe antibiotics, chemical compounds, and also small molecules from plant sources. Of the 150 unique compounds, 101 compounds are FDA approved. We selected the top 20 FDA-approved molecules based on safety and potency and studied their minimum inhibitory concentration and minimum bactericidal concentration. The promising safe FDA-approved candidates that show low minimum inhibitory concentration and minimum bactericidal concentration values can be chosen as lead molecules for further advanced studies.
\end{abstract}

Keywords: Lyme disease, Borrelia burgdorferi, BacTiter-Glo assay, high-throughput screening, persisters

\section{Introduction}

Lyme borreliosis, commonly referred to as Lyme disease, is the most common zoonotic bacterial disease in North America. It is estimated $>300,000$ cases per annum in USA are reported. ${ }^{1}$ Lyme disease is caused by the spirochetes of genus Borrelia, collectively known as Borrelia burgdorferi sensu lato. Among the genus, B. burgdorferi sensu stricto (B. burgdorferi s.s.) is the single major causative agent of the disease in USA. Other members in the group include Borrelia duttonii, Borrelia garinii, Borrelia afzelii, and Borrelia miyamotoi, which are involved in infections in Europe, Eurasia, and other parts of the world..$^{2,3}$ Important clinical presentations of Lyme disease in humans include erythema migrans, fatigue, fever, chills, muscle, and joint pain. ${ }^{4,5}$ Approximately $10 \%-20 \%$ of the patients who have been treated with antibiotic therapy reported the 
reappearance of symptoms, such as muscle and joint pain and generalized fatigue. In some patients, these symptoms last for $>6$ months after the initial treatment. Several researchers categorize this condition as posttreatment Lyme disease syndrome (PTLDS), and it is observed in patients even after rigorous antibiotic treatment. ${ }^{6}$ However, the reason for the reoccurrence of these symptoms in some patients is unclear. This might be due to the hyperresponsive immune reaction to the deposited antigenic debris of the Borrelia or any drugtolerant persisters. ${ }^{7,8}$ Some researchers have raised the question that Borrelia may persist in some hosts after antibiotic treatment, but the idea is controversial. .,10 $^{9}$

In the absence of antibiotic therapy, B. burgdorferi can affect immunocompetent host and can establish long-term infections lasting years to lifelong in its natural (ie, mice) and incidental (ie, humans) hosts. ${ }^{11,12}$ Although current animal models (mice, dogs, and rhesus macaque monkeys) do not completely mimic human infection, studies have shown that antibiotic therapy with doxycycline, ceftriaxone, or tigecycline did not fully eradicate $B$. burgdorferi, as determined by xenodiagnosis method. ${ }^{13-16}$ Many researchers have expressed their concerns on how the previously mentioned studies were conducted. Recent studies in mice showed a resurgence of B. burgdorferi after 12 months of treatment with antibiotics. Though Borrelia was not found when cultured, the RNA transcripts for multiple genes were detected. ${ }^{15}$ In a recent study, B. burgdorferi DNA was identified by xenodiagnosis in a human PTLDS patient despite antibiotic treatment. ${ }^{17}$

Three morphological forms of persistent B. burgdorferi were reported based on the observations from the experimental studies. They are spirochete, spheroplast (or L-form), microcolony, and round-body forms. ${ }^{10,18,19}$ Though there are some reports of spheroplast or round-body forms in humans, there is no clear study demonstrating whether these forms exist and cause clinical disease in humans. ${ }^{20}$ Recently, few systematic studies showed the presence of drug-tolerant B. burgdorferi persisters during in vitro culture. Sharma et $\mathrm{al}^{21}$ have demonstrated that killing of $B$. burgdorferi by antibiotics is biphasic, with a small subpopulation of surviving persisters. This is one of the systematic studies that has revealed the existence of Borrelia persisters in vitro. In addition to this study, researchers showed the presence of antibiotic-tolerant persisters. Researchers have shown that the combination of three drugs, such as daptomycin, doxycycline, and cefoperazone, eradicated the most resistant microcolony form of $B$. burgdorferi persisters. ${ }^{7,22}$ The currently prescribed drugs amoxicillin, ceftriaxone, and doxycycline are not able to eliminate the $B$. burgdorferi completely according to some studies, ${ }^{7,21-23}$ although other investigators have shown the complete eradication of B. burgdorferi both in vitro and in vivo after relatively low dose or short course treatment with antibiotics. ${ }^{24-26}$ The surviving antibiotic-tolerant cells are not resistant mutants; upon regrowth, they form a new persister subpopulation. So, based on these observations, it is necessary to identify the potent drug candidates that can target the Borrelia persisters.

Due to the unmet need, studies using high-throughput screening (HTS) of drugs for B. burgdorferi are increasing, especially screening of drugs against persisters. ${ }^{7,27-30}$ HTS of medicinal compound libraries can help in identifying candidate molecules that can potentially be effective against the persisters and, therefore, can be used for therapeutic purposes. Recent studies of a number of compounds have facilitated the repurposing of drug molecules and their rapid approval for the treatment of different diseased conditions. ${ }^{31,32}$ Moreover, the availability of structurally diverse entities in the HTS compound libraries can help in identifying the potential lead compounds that can be used for the antiborrelial drug discovery process. The current study was, therefore, aimed at testing multiple compound libraries for their potential to inhibit the growth of stationary-phase B. burgdorferi. Four drug-screening libraries were selected on the basis of the available pre/clinical data, structural diversity, and mechanism of action of the compounds. Using recently developed highly sensitive BacTiter-Glo assay, four chemical libraries, such as the Library of Pharmacologically Active Compounds (LOPAC1280), the National Institutes of Health Clinical Collection (NIHCC), the Microsource Spectrum, and the Biomol Food and Drug Administration (FDA), were screened. ${ }^{33}$ We found 150 inhibitory compounds that were found to be active against the stationary-phase form of Borrelia. The minimum inhibitory concentration (MIC) and minimum bactericidal concentration $(\mathrm{MBC})$ were determined for the top 20 FDA-approved compounds. The identified compounds can be further investigated for their therapeutic potential in preclinical animal models and in patients with Lyme disease. Moreover, the outcomes of the study can provide input for both mechanistic and translational research that can help in establishing clinically viable solutions to Lyme disease.

\section{Materials and methods Bacterial strains and culture}

B. burgdorferi s.s. strains CA4 and CA8 originated biologically from Ixodes pacificus ticks, USA. The bacterial strains were generously provided by Dr Robert Lane, University of California, Berkeley, CA, USA. These strains are infectious low passage numbers that were cultured in Barbour-Stoenner-Kelly II (BSK-II) complete medium, with $6 \%$ rabbit serum (Sigma-Aldrich, St Louis, MO, USA). 
We have chosen these strains because these are infectious and prevalent in California, USA. The cultures were grown in sterile $50 \mathrm{~mL}$ Falcon tubes (Corning Incorporated, Corning, NY, USA) and incubated at $33^{\circ} \mathrm{C}$. All culture media were sterilized with $0.2 \mu \mathrm{M}$ filter units (EMD Millipore, Billerica, MA, USA). The B. burgdorferi cultures were grown for 7-10 days to reach the stationary phase with cell density $>10^{8} / \mathrm{mL}$ for performing all the assays. For HTS drug screening, 7-10-day-old stationary-phase B. burgdorferi cultures were transferred to 384-well culture microplates.

\section{Drugs and drug libraries}

All the information regarding purchase, solubility, and stock solutions of drugs used in this study have been provided in Table 1. All the drug stocks were filter sterilized with $0.2 \mu \mathrm{M}$ filter units. The FDA-approved drug libraries, such as the LOPAC1280, the NIHCC, the Microsource Spectrum, and the Biomol FDA (now Enzo Life Sciences) were acquired from High-Throughput Bioscience Center, Stanford University. All the library stocks were maintained in dimethyl sulfoxide solutions at $10 \mathrm{mM}$ compound concentrations. Plate-to-plate dilutions were performed in 384-well plates using an Evolution P3 system equipped with a 384-well head.

\section{HTS of chemical libraries with B. burgdorferi persisters}

To identify the effect of chemical compounds on B. burgdorferi stationary-phase cultures qualitatively, HTS was performed using the following procedure. A total of $50 \mu \mathrm{L}$ of BSK-II medium was added to white 384-well Corning plates (Corning Incorporated) using the Matrix Wellmate, and $\sim 100 \mathrm{~nL}$ of each compound from the stock solution was added using the pin tool in the Staccato System (CaliperLS, Caliper Life Sciences Inc, Alameda, CA) to 1-22 columns. The last two columns of the 384 wells were left for culturing the controls. We screened one compound per well in a 384-well microplate format. These are screened in a 7-point titration ranging from $25 \mu \mathrm{M}, 12.5 \mu \mathrm{M}, 7.25 \mu \mathrm{M}, 3.625 \mu \mathrm{M}, 1.81 \mu \mathrm{M}, 0.9 \mu \mathrm{M}$, and $0.45 \mu \mathrm{M}$. To these plates, $25 \mu \mathrm{L}$ of $10^{6} / \mathrm{mL}$ B. burgdorferi stationary-phase cultures were added using the Multidrop dispenser. Then the plates were incubated at $33^{\circ} \mathrm{C}$ for 96 hours in a humidified $\mathrm{CO}_{2}$ incubator (Forma Scientific, Waltham, MA, USA) having $5 \% \mathrm{CO}_{2}$ and $95 \%$ air. After 96 hours, $25 \mu \mathrm{L}$ of BacTiter-Glo reagent (Promega Corporation, Fitchburg, WI, USA) was added to the plates using the Multidrop dispenser. The plates were shaken for 2 minutes and incubated at room temperature for 5 minutes. Finally, the luminescence was measured using a Flexstation 3 (Molecular Devices LLC, Sunnyvale, CA, USA) (500 ms/well). The data were analyzed using the Assay Explorer software. Hits were identified as compounds that resulted in a decrease in the luciferase signal compared to controls with no compound.

\section{Determination of MIC and MBC}

The MIC of the small molecules identified through screening was determined by culturing $10^{6} / \mathrm{mL}$ Borrelia in BSK-II medium with different concentrations $(0.3-160 \mu \mathrm{M})$ of drugs. For MIC, $1 \mathrm{~mL}$ of cultures with respective drugs are

Table I Details of drugs used

\begin{tabular}{|c|c|c|c|c|}
\hline Serial number & Name & Supplier & Solubility & Stock solution (mM) \\
\hline I & Tetraethylthiuram disulfide & Cayman Chemical & DMSO & 10 \\
\hline 2 & Doxorubicin hydrochloride & Cayman Chemical & Water & 10 \\
\hline 3 & Josamycin & Sigma-Aldrich & Ethanol & 20 \\
\hline 4 & Cefotaxime acid & Cayman Chemical & DMSO & 20 \\
\hline 5 & Cefazolin sodium & Cayman Chemical & Water & 10 \\
\hline 6 & Epirubicin hydrochloride & Cayman Chemical & Water & 10 \\
\hline 7 & Erythromycin ethylsuccinate & Santa Cruz Biotech & DMSO & 20 \\
\hline 8 & A-23 I87 calcimycin & Cayman Chemical & DMSO & 10 \\
\hline 9 & Gramicidin & Sigma-Aldrich & DMSO & 20 \\
\hline 10 & Cefdinir & Sigma-Aldrich & DMSO & 20 \\
\hline II & Gambogic acid & Cayman Chemical & DMSO & 20 \\
\hline 12 & Cephalothin sodium & Santa Cruz Biotech & Water & 10 \\
\hline 13 & Ceftazidime & Cayman Chemical & DMSO & 5 \\
\hline 14 & Ticarcillin disodium & Santa Cruz Biotech & Water & 20 \\
\hline 15 & Valinomycin & Cayman Chemical & Ethanol & 20 \\
\hline 16 & Moxifloxacin hydrochloride & Santa Cruz Biotech & Water & 20 \\
\hline 17 & Linezolide & Santa Cruz Biotech & Water & 15 \\
\hline 18 & Idarubicin $\mathrm{HCl}$ & Sigma-Aldrich & Water & 20 \\
\hline 19 & Tosufloxacin tosylate & Santa Cruz Biotech & DMSO & 10 \\
\hline 20 & Azlocillin sodium & Santa Cruz Biotech & Water & 10 \\
\hline
\end{tabular}

Abbreviation: DMSO, dimethyl sulfoxide. 
grown in 48-well plates in triplicates, wrapped with paraffin film and placed for 72 hours at $33^{\circ} \mathrm{C}$ in a humidified $\mathrm{CO}_{2}$ incubator (Forma Scientific) having 5\% $\mathrm{CO}_{2}$ and $95 \%$ air. ${ }^{23}$ Cell proliferation was assessed using the bacterial counting chamber (Petroff-Hausser Counter, Horsham, PA, USA) by phase-contrast microscopy. At the same time, cell proliferation was also assessed using the BacTiter-Glo ${ }^{\mathrm{TM}}$ assay. The counting was performed in all the 25 squares of the central grid. The BacTiter-Glo ${ }^{\mathrm{TM}}$ assay is performed by mixing $100 \mu \mathrm{L}$ of culture in each well with $100 \mu \mathrm{L}$ of BacTiter-Glo ${ }^{\mathrm{TM}}$ reagent (Promega Corporation). Then, the assay is performed according to the manufacturer's instructions. Luminescence was measured using a Flexstation 3 microplate reader at

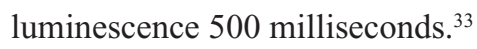

For determining MBC, $20 \mu \mathrm{L}$ of the $10^{6} / \mathrm{mL}$ Borrelia cultures grown in BSK-II medium for 72 hours at different drug concentrations were added to the fresh BSK-II medium and subcultured for 3 weeks. ${ }^{23,34-36}$ After a 3 week incubation period, the samples were observed microscopically for motile spirochetes in the culture. The MIC and MBC determinations were done thrice independently.

\section{Time kill studies}

Time kill studies were performed with borrelial isolate CA8 (B. burgdorferi s.s.) with azlocillin sodium and cefotaxime acid. To determine the rate of antimicrobial activity, $10^{6} / \mathrm{mL}$ Borrelia cultures were grown in BSK-II medium with drugs at different concentrations. BSK-II medium with no drugs was used as a control. The antibacterial activity was determined by counting bacteria at 24 hours, 48 hours, and 72 hours. The experiment was done once with triplicates. ${ }^{34}$

\section{Result}

\section{Development of BacTiter-Glo ${ }^{\mathrm{TM}}$ assay compatible to HTS}

B. burgdorferi grows very slowly, typically taking from 12 hours to 18 hours to replicate. Due to this slow growth, it is difficult to measure $B$. burgdorferi culture quantitatively using direct optical density. To measure the bacterial viability and antibiotic susceptibility of $B$. burgdorferi, several rapid colorimetric assays, such as fluorogenic dye SYTO 9 (LIVE/DEAD) assay, Sytox Green/Hoechst 33342 assay, and SYBR Green I/ PI assay, have been developed. 7,28,37 It was reported that SYBR Green I/PI assay can only detect accurately the live/dead ratio of $10^{5}$ cells $/ \mathrm{mL} .{ }^{37}$ In parallel to these assays, we have developed a highly sensitive BacTiter-Glo ${ }^{\mathrm{TM}}$ assay, which has been optimized for 384-well plate. ${ }^{33}$ It is a one-step, straightforward method to assess bacterial viability by measuring ATP from the given sample. As it is a single-step assay, BacTiter-Glo ${ }^{\mathrm{TM}}$ assay can screen drugs quickly and efficiently in a large scale. We have recently reported that the BacTiter-Glo ${ }^{\mathrm{TM}}$ assay is a very sensitive assay that can reliably detect signal in the range of minimum ten Borrelia cells in phosphate-buffered saline and $7 \times 10^{3}$ in BSK-II medium. ${ }^{33}$ The BacTiter-Glo ${ }^{\mathrm{TM}}$ assay can only detect cells that can generate ATP. It has not been tested whether BacTiter-Glo ${ }^{\mathrm{TM}}$ assay can detect nongrowing Borrelia, which may produce low levels of ATP. Due to the advantage of detecting low number of bacteria $\left(7 \times 10^{3}\right)$ by this method, false-positive candidates are eliminated in the HTS. The BacTiter-Glo assay was validated by calculating $Z^{\prime}$ values with the Assay Explorer software for CA4 and CA8 strains, which were $>0.6$, considered to be a good value to perform an HTS assay. The data indicate that the BacTiter-Glo ${ }^{\text {TM }}$ assay provides a one-step, straightforward method to quantify B. burgdorferi with good sensitivity and dynamic range.

\section{High-throughput primary screening of chemical libraries with B. burgdorferi persisters to identify potent drugs}

To identify safe and effective molecules for Lyme disease treatment, repurposing FDA-approved drug molecules might be a fast and viable alternative in developing novel borrelicidal compounds. To achieve this, we utilized the BacTiter-Glo ${ }^{\mathrm{TM}}$ assay system. All the screening of drugs was done by stationary-phase CA8 borrelial cultures with cell density $>10^{8} / \mathrm{mL}$, which are grown in BSK-II medium for 7-10 days. During screening, the last two rows in the 384-well plate were taken as control by not adding any drugs. By using the HTS platform, we have screened 4,366 chemical compounds representing different libraries, including the Sigma LOPAC (1,280 compounds), the NIHCC (446 compounds), the Microsource Spectrum (2,000 compounds), and the Biomol FDA (640 compounds). The screening was repeated for seven times with a titration range from $0.45 \mu \mathrm{M}$ to $25 \mu \mathrm{M}$ to confirm the reproducibility. That means each drug is tested at different concentrations in seven different plates. By successfully screening these libraries, we have identified $\sim 150$ hit molecules that showed the inhibition of bacterial growth $>90 \%$ compared to the control. Out of these 150 hit molecules, 101 (67.3\%) small molecules are FDA-approved compounds.

Based on the results from the primary screening, we chose the top 20 candidates and reconfirmed these by secondary screening with BacTiter-Glo ${ }^{\mathrm{TM}}$ assay. These candidates were chosen based on the $>95 \%$ inhibition of bacteria in primary screening, FDA approval, and safety of the compounds (Table 2). The doxycycline, which is one of the currently 
Table 2 Structure and activity of Top 20 hits against B. burgdorferi

\begin{tabular}{|c|c|c|c|c|c|}
\hline $\begin{array}{l}\text { Serial } \\
\text { number }\end{array}$ & Drugs & Structure & $\%$ inhibition & $\begin{array}{l}\text { MIC } \\
(\mu \mathrm{M})\end{array}$ & $\begin{array}{l}\text { MBC } \\
(\mu M)\end{array}$ \\
\hline & Control (no drug) & - & 0 & & \\
\hline & Doxycycline & - & 94.14 & & \\
\hline I & $\begin{array}{l}\text { Tetraethylthiuram } \\
\text { disulfide }\end{array}$ & & 99.80 & 0.625 & 1.25 \\
\hline 2 & $\begin{array}{l}\text { Doxorubicin } \\
\text { hydrochloride }\end{array}$ & & 99.70 & 0.625 & 1.25 \\
\hline 3 & Josamycin & & 99.63 & 15.0 & 20.0 \\
\hline 4 & Cefotaxime acid & & 99.47 & 2.0 & 3.0 \\
\hline 5 & Cefazolin sodium & & 99.20 & 1.25 & 12 \\
\hline 6 & $\begin{array}{l}\text { Epirubicin } \\
\text { hydrochloride }\end{array}$ & & 99.10 & 0.3 & 0.625 \\
\hline 7 & Erythromycin & & 99.04 & 15.0 & 20.0 \\
\hline
\end{tabular}


Table 2 (Continued)

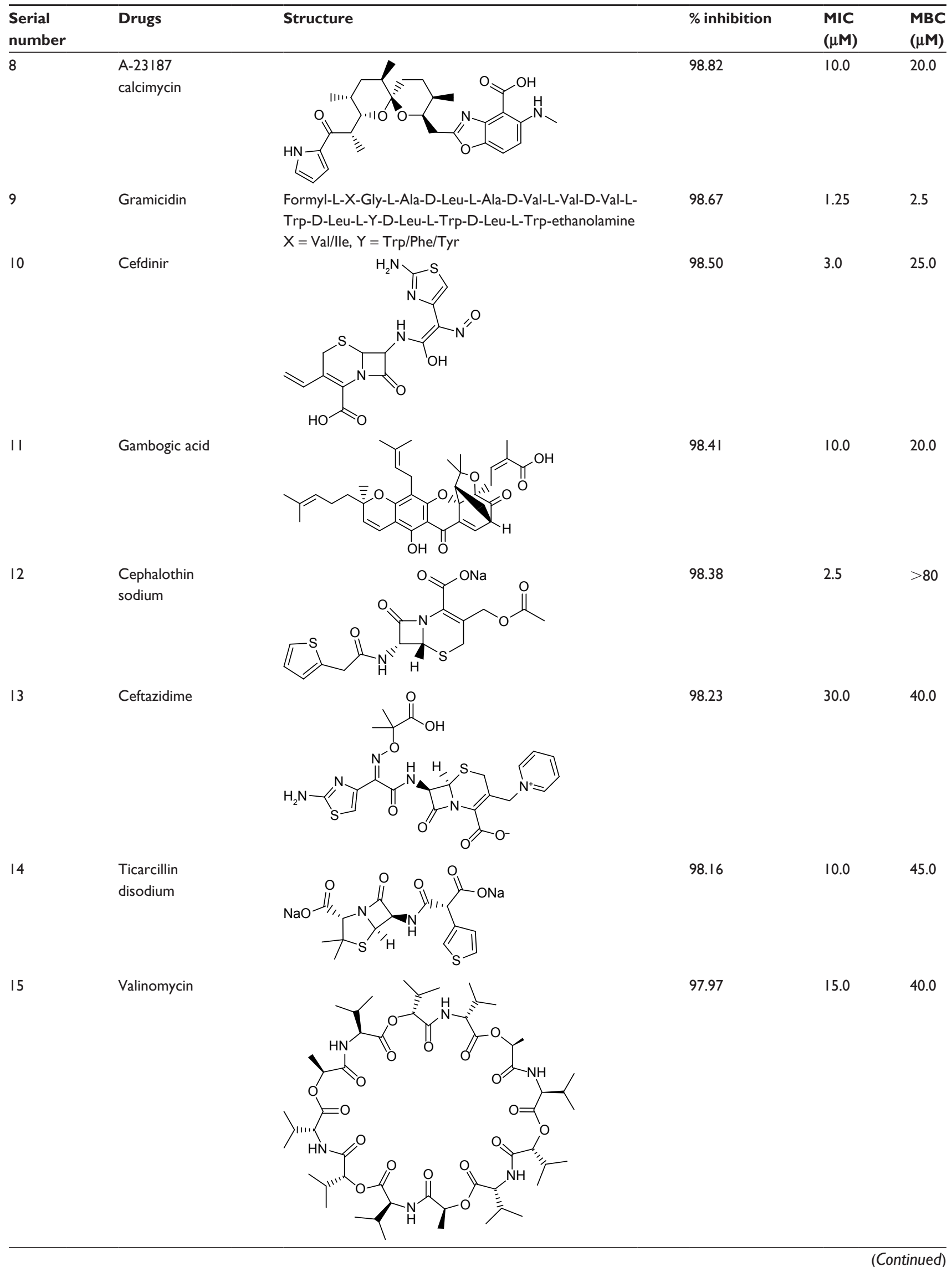


Table 2 (Continued)

\begin{tabular}{|c|c|c|c|c|c|}
\hline $\begin{array}{l}\text { Serial } \\
\text { number }\end{array}$ & Drugs & Structure & $\%$ inhibition & $\begin{array}{l}\text { MIC } \\
(\mu \mathrm{M})\end{array}$ & $\begin{array}{l}\text { MBC } \\
(\mu \mathrm{M})\end{array}$ \\
\hline 16 & $\begin{array}{l}\text { Moxifloxacin } \\
\text { hydrochloride }\end{array}$ & & 97.68 & 7.5 & 12.5 \\
\hline 17 & Linezolid & & 97.58 & 0.625 & 25.0 \\
\hline 18 & $\begin{array}{l}\text { Idarubicin } \\
\text { hydrochloride }\end{array}$ & & 97.4 & 5.0 & 10.0 \\
\hline 19 & $\begin{array}{l}\text { Tosufloxacin } \\
\text { tosylate }\end{array}$ & & 97.27 & 45.0 & $>80$ \\
\hline 20 & Azlocillin sodium & & 95.25 & 1.25 & 2.5 \\
\hline
\end{tabular}

Abbreviations: MBC, minimum bactericidal concentration; MIC, minimum inhibitory concentration.

prescribed drugs inhibited $94.14 \%$ of borrelial growth compared to the control (no drug), which is less than the percentage of inhibition of the top 20 candidates (Table 2). Percentage inhibition was also one of the criteria for selecting top 20 candidates for testing. The results of the remaining 130 compounds are shown in Table S1. Of the Borrelia cultured with some of the drugs, the viability was evaluated with BacTiter-Glo ${ }^{\mathrm{TM}}$ assay as shown in Figure 1. In Figure 1A, tetraethylthiuram disulfide shows the complete borrelial cell inhibition at $1.25 \mu \mathrm{M}$, while epirubicin hydrochloride and doxorubicin hydrochloride are uniformly effective against B. burgdorferi at $0.625 \mu \mathrm{M}$. Vehicle control (dimethyl sulfoxide) did not show any significant effect on the cell survival.
Drugs azlocillin and cephalothin showed $\sim 99 \%$ efficacy at $0.31 \mu \mathrm{M}$ (Figure 1B). After confirming from secondary screening, the MIC and MBC values were determined by microdilution method for all the potential candidates.

\section{Determinations of $M I C$ and $M B C$ values}

To confirm the efficacy of the screening, MIC and MBC values were evaluated. The MIC is determined as the lowest concentration at which no motile spirochete is observed by microscopy. Of the top 20 candidates evaluated, the MIC values of epirubicin hydrochloride and doxorubicin hydrochloride are $<1 \mu \mathrm{M}$. For the drugs cefazolin sodium, gramicidin, azlocillin sodium, cefotaxime, and cephalothin 
A

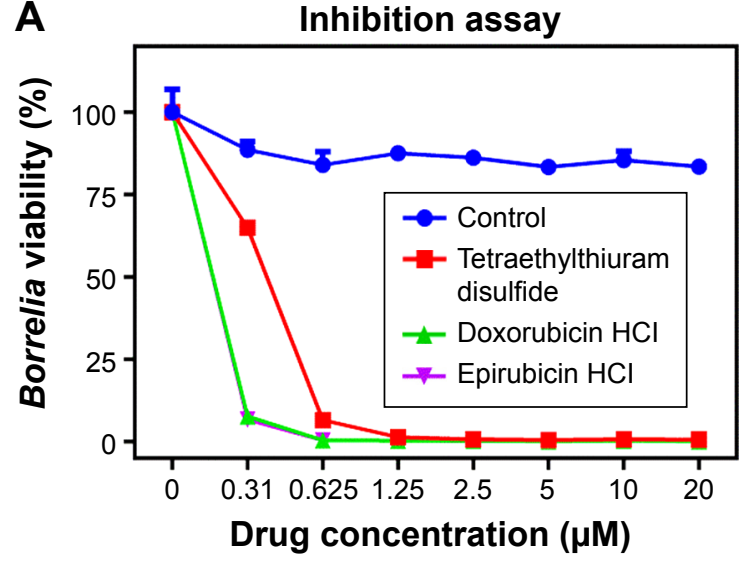

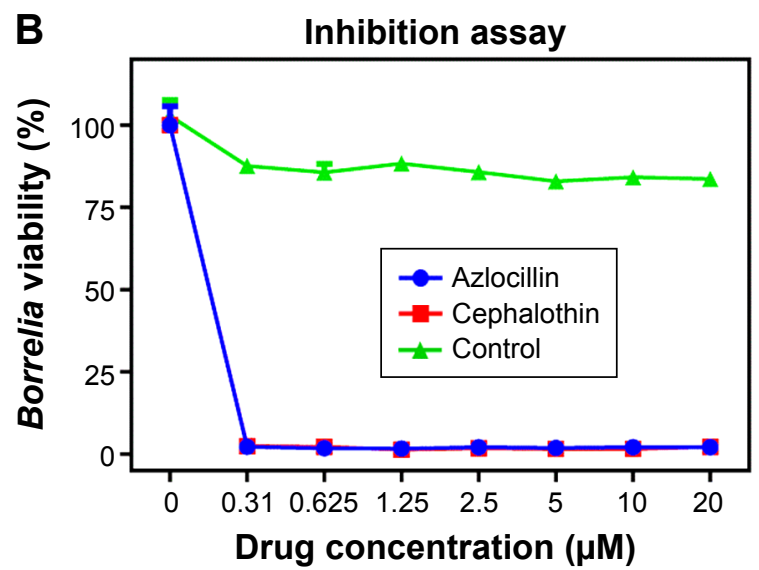

Figure I Inhibition assay of drugs on CA8 strain.

Notes: Effect of drugs on Borrelia cell viability was studied with drugs: (A) tetraethylthiuram disulfide, doxorubicin hydrochloride, and epirubicin hydrochloride and (B) azlocillin sodium and cephalothin sodium. The control has no drugs. The results represent mean \pm SD.

Abbreviation: SD, standard deviation.

sodium, tetraethylthiuram disulfide, and linezolid, the MIC values are $\leq 3 \mu \mathrm{M}$. For the remaining drugs, the MIC values are $\geq 3 \mu \mathrm{M}$. The $\mathrm{MBC}$ was determined by subculturing $20 \mu \mathrm{L}$ of the Borrelia cultures grown at different drug concentrations in fresh BSK-II medium for 21 days. ${ }^{23,34}$ The MBC was determined when no spirochete was observed microscopically in the culture. Of the top 20 compounds, the lowest MBC values $(<1.5 \mu \mathrm{M})$ were observed for epirubicin hydrochloride and doxorubicin hydrochloride. The drugs gramicidin, azlocillin sodium, leucomycin, cefotaxime, idarubicin, and tetraethylthiuram disulfide show the $\mathrm{MBC}$ values of $\leq 10 \mu \mathrm{M}$. All the remaining drugs show the $\mathrm{MBC}$ values of $>10 \mu \mathrm{M}$. For the cephalothin sodium, the MIC

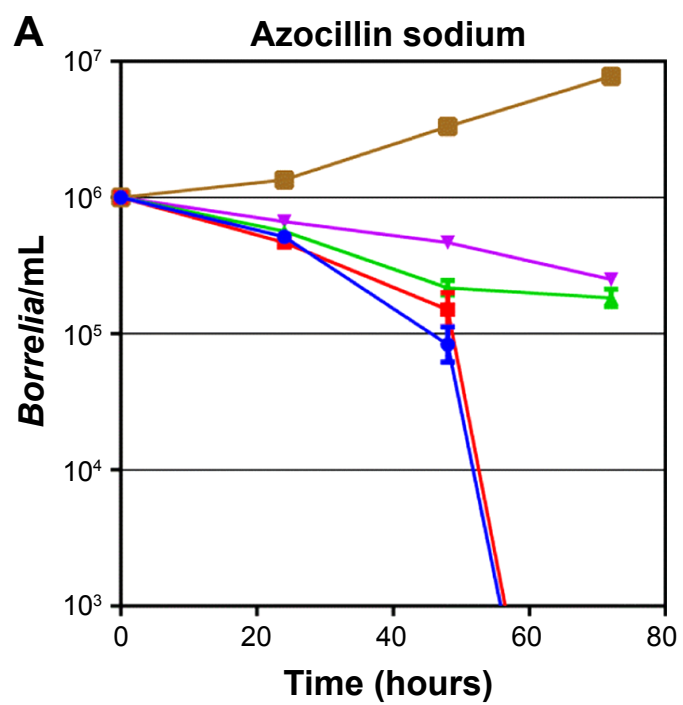

value is very low, but the MBC value is $>80 \mu \mathrm{M}$, and for tosufloxacin tosylate, both the MIC and MBC values are very high. The compounds doxorubicin, cephalothin, ticarcillin, and cefdinir were also reported in the HTS performed by Feng et al. ${ }^{29,38}$

\section{Time kill studies}

To determine the rate of antimicrobial activity of azlocillin sodium and cefotaxime acid, CA8 strain of Borrelia was exposed to different concentrations $(0.625 \mu \mathrm{M}, 1.25 \mu \mathrm{M}$, $2.5 \mu \mathrm{M}$, and $5 \mu \mathrm{M}$ ) of each of the drugs. The initial Borrelia inoculum did not even decrease $1-\log _{10}$-unit at concentrations $0.625 \mu \mathrm{M}$ and $1.25 \mu \mathrm{M}$ for azlocillin sodium (Figure $2 \mathrm{~A}$ ).

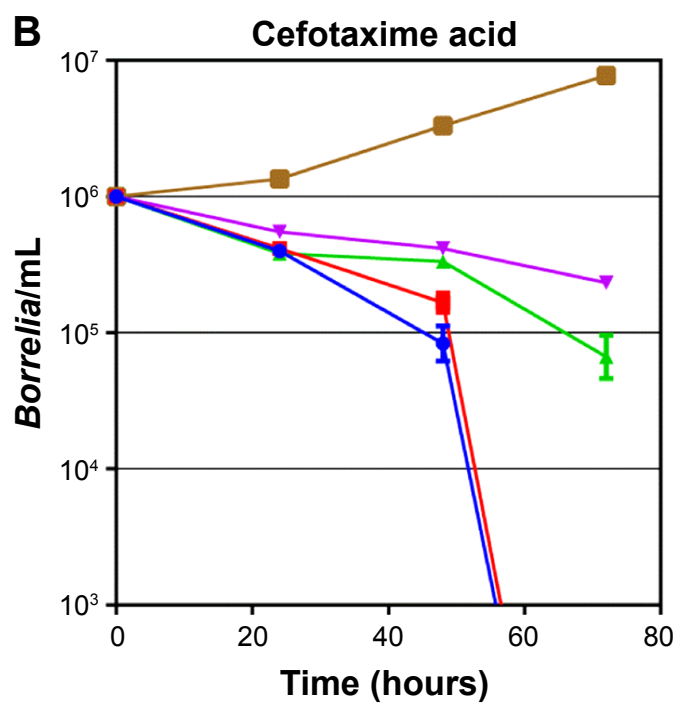

Control

Figure 2 Time kill curves for B. burgdorferi s.s. isolates CA8 with (A) azlocillin sodium and (B) cefotaxime acid.

Notes: The Borrelia was grown in the drug concentrations of $0.625 \mu \mathrm{M}, 1.25 \mu \mathrm{M}, 2.5 \mu \mathrm{M}$, and $5 \mu \mathrm{M}$. Experiment was performed with triplicates by the investigation of growth using conventional cell counts, and data were reported as the mean of triplicate. The control has no drugs.

Abbreviation: B. burgdorferi, Borrelia burgdorferi. 
For cefotaxime acid at concentration $1.25 \mu \mathrm{M}$, the microbial growth is decreased 1-log10-unit but not at $0.625 \mu \mathrm{M}$ (Figure 2B). Both azlocillin sodium and cefotaxime acid reduced morphologically intact motile cells to 3-log 10 -unit (99.9\%) between 48 hours and 72 hours at concentrations $2.5 \mu \mathrm{M}$ and $5 \mu \mathrm{M}$. These specified concentrations of $2.5 \mu \mathrm{M}$ and $5 \mu \mathrm{M}$ are MIC values and twice the MIC values. In control, the Borrelia growth increased to 7-log10-unit.

\section{Discussion}

One of the major challenges in the discovery of potent therapeutically effective drug molecules is the lack of availability of sensitive screening tools. Though the procedure becomes fairly simple with the availability of HTS methods and large collections of drug libraries, the development of drugs against slow-growing organisms still remains an uphill task. Though a number of drugs have been tested and approved against various spirochetes, the currently available drug therapies, such as amoxicillin and doxycyclin, was unable to kill $B$. burgdorferi persisters in vitro. ${ }^{7,21}$ In addition to this, patients with Lyme who are diagnosed later and treated may develop arthritis. These patients treated later do not respond fully to a first course of antibiotic therapy. ${ }^{21,39}$ Therefore, efforts need to be made to develop an efficient platform for the screening of small molecules that can prove to be very effective, yet safe, in the treatment of Lyme disease.

In an effort to search for the lead molecules that can potentially be used for clinical application, we developed and used a faster, efficient, and reliable HTS platform for the screening of drug molecules against Borrelia. Current methods are not sensitive enough to detect the bacterial counts of $<10^{5} / \mathrm{mL} .{ }^{37}$ Due to this limitation, many potentially valuable hits would be lost during the compound-screening process. To avoid this, we developed a robust BacTiter-Glo assay. We exploited the dependence of B. burgdorferi on ATP as the major determinant of cell viability and their metabolic activity by utilizing BacTiter-Glo assay. ${ }^{33}$ This assay involves the addition of an active reagent that binds with ATP molecules and releases a luminescent signal, indicating the presence of the viable microbes in the medium. In order to develop a faster, yet reliable assay, we optimized the assay for a 384-well HTS format. ${ }^{33}$

After developing and optimizing the assay, we focused on analyzing a diverse group of chemical compounds and drug libraries in order to identify the most effective agents that can inhibit the growth of Borrelia. For this purpose, we screened four different compound libraries that had a greater proportion $(80 \%)$ of FDA-approved molecules in them. Approximately 4,366 drug molecules belonging to four different libraries, including the Sigma LOPAC, the NIHCC, the Microsource Spectrum, and the Biomol FDA, were screened. Of the four chemical libraries, except for the Microsource Spectrum, all the other libraries are 100\% FDA approved. The compounds included in these libraries varied in their chemical structures, pharmacological classes, mechanisms of therapeutic action, and their origins. The rationale for selecting such a diverse group was to ensure that the identified compounds could find direct clinical applications or provide basis for lead compounds for drug design and development process.

We then exploited the developed HTS platform to identify the hit molecules that showed the inhibition of $>90 \%$ based on the bacterial cell viability. A total of 150 molecules, both FDA approved and unapproved, were shortlisted from the different libraries. However, the utilization of approved drug molecules helps in shortening the timeline from bringing the drug from the lab to the bedside. Moreover, the availability of clinical pharmacological and toxicological data decreases the overall cost of the drug discovery process. Therefore, out of the obtained HTS hits, we selected 20 molecules based upon their FDA approval status, MIC values $(<20 \mu \mathrm{M})$, percentage inhibition of stationary-phase cultures of Borrelia $(>95 \%)$, and their reported safety profiles. The list of compounds that has been identified after an additional secondary screen (BacTiter-Glo), microscopic evaluation, and MIC and MBC determination is provided in Table 2 . It was found that the identified compounds have been reported to exploit a number of mechanisms to exhibit their pharmacological action in other bacterial species. The identification of compounds using multiple pathways for their borrelicidal effects provides an opportunity for using these drugs in clinical setup as combination therapy or possible therapeutic equivalents.

Among the identified drug molecules, erythromycin and kitasamycin appeared to be very active. The drug erythromycin was already used clinically for the treatment of Lyme disease. In some laboratories, strains and clinical isolates of B. burgdorferi show resistance to erythromycin. ${ }^{40}$ This drug belongs to the macrolide group of antibiotics, which exhibit their antibacterial potential by blocking the protein synthesis assembly of the bacteria. By targeting peptidyltransferase of the bacteria, macrolides tend to cause the termination of protein chain elongation and the detachment of the enzyme from the ribosomes, resulting in the bacterial protein inhibition. ${ }^{41}$ Moreover, the drug molecules have also proved their effectiveness as immune modulators in the treatment of various inflammatory conditions. ${ }^{42,43}$ The drugs, erythromycin and kitasamycin, are readily absorbed, and they diffuse into most of the body fluids. ${ }^{44-46}$ Additionally, their biochemical 
features make them extremely capable for intracellular and tissue penetration. The availability of the antibiotics in the drug-sequestered sites can potentially be helpful in clearing bacteria from skin and joints. ${ }^{47-49}$ Additionally, a high percentage inhibition of stationary-phase culture forms of the bacteria and the low MIC and MBC values establish that these drug molecules can be helpful in treating both dividing and persistent forms of Borrelia.

One of the other drug molecules, linezolid, was found to possess $\sim 98 \%$ inhibition of the stationary-phase Borrelia in the initial screen. Moreover, the drug was found to possess the MIC and MBC values of $0.4 \mu \mathrm{g} / \mathrm{mL}$ and $8.4 \mu \mathrm{g} / \mathrm{mL}$, respectively. The probable mechanism of action of linezolid is also by retarding the initiation step of protein translation. It tends to bind to the active site of the $50 \mathrm{~S}$ ribosomal assembly and prevents the downstream translation process, resulting in the termination of normal protein synthesis in the bacteria. ${ }^{44,47}$ Linezolid possesses commendable pharmacokinetic profile with up to $100 \%$ bioavailability after oral administration and has a high volume of distribution. ${ }^{48,49}$ It is efficiently distributed in most of the body tissues, including osteoarticular and central nervous system. ${ }^{50,51}$ Another group of compounds that demonstrated significant reduction in bacterial growth included the anthracycline-based antitumor antibiotics, ie, epirubicin, doxorubicin, and idarubicin. These drugs, primarily used for treating cancer conditions, tend to nonselectively intercalate between the nucleotides and prevent DNA replication. Moreover, they inhibit the topoisomerase II (bacterial equivalent DNA gyrase) and prevent the relaxation of DNA supercoiling, leading to decreased replication and transcription processes. ${ }^{52}$ Additionally, these drugs cause genome and transcriptome level damage by causing the generation of free oxygen radicals and disassembly of histone proteins. ${ }^{53}$ All these anthracycline antibiotics possess exceptional pharmacokinetic profile. Though these molecules are very effective as cytotoxic agents for the treatment of cancer conditions, their use is often limited by their ability to cause cardiotoxicity, neutropenia, and other adverse drug reactions. ${ }^{54}$ Modifications in the formulation or developing safer therapeutic drug molecules based on the anthracycline ring can be helpful in finding acceptable alternate strategies against the drug-tolerant Borrelia persisters. ${ }^{7}$

Moxifloxacin, a fourth-generation fluoroquinolone, also showed a good inhibitory effect on the stationary-phase cultures of Borrelia. The drug specifically targets DNA gyrase, both topoisomerase II and topoisomerase IV, and causes the cessation of uncoiling and the separation of double-stranded DNA, resulting in the inhibition of bacterial replication process. ${ }^{55}$ Moxifloxacin, also available in the oral formulation, has $>80 \%$ bioavailability. The drug has been known to target various bacteria in the tissues that are generally considered as drug-sequestered sites, including brain, lungs, and bones. ${ }^{56} \mathrm{It}$ has also been proved to be up to ten times more potent and effective in treating other intracellular bacteria involved in latent infections, such as Mycobacterium tuberculosis, than other drugs of the same group. ${ }^{57}$ Another fluoroquinolone, tosufloxacin, also showed comparable inhibitory activity of the borrelial stationary-phase cultures ( $>97 \%$ inhibition). However, the MIC and MBC values of the compound were greater than moxifloxacin. The drug also targets the DNA gyrase and has appreciable pharmacokinetic properties. ${ }^{58}$ Based on these pharmacological properties, moxifloxacin and tosufloxacin appear to be a safe and effective alternate for the currently available amoxicillin- or doxycycline-based antiborrelial therapeutic strategies.

The study also identified a number of drug molecules that target the cell wall synthesis of bacterial species. Among these, the parenteral antipseudomonal penicillins, azlocillin and ticarcillin, were found to be quite potent against the stationary-phase and growing forms of B. burgdorferi. These penicillins tend to target the transpeptidase enzyme of the bacteria, resulting in the inability of the growing peptidoglycan layer of the cell wall to cross-link. ${ }^{59}$ Penicillins, bearing a smaller molecular structure, tend to pass through the cell wall and inhibit the enzyme. This leads to the generation of spheroplast or cell wall-lacking forms of the bacteria, which are highly susceptible to cytolysis and cell death. Being $\beta$-lactam antibiotics, these drug molecules are highly susceptible to the action of $\beta$-lactamase-like enzymes. However, the use of aminoglycosides or clavulanates in the antibiotic regimen has proved to be effective in overcoming the issues of drug resistance.

Using our HTS method, we were also able to identify some cephalosporins that were very active against the persistent forms of Borrelia. Cephalothin, cefdinir, cefotaxime acid, and ceftazidime had a bacterial inhibition value of $>98 \%$. Cephalosporins follow a mechanism similar to penicillins for demonstrating their antibacterial effects. However, these drugs are more resistant to the action of $\beta$-lactamases. Cephalothin, cefotaxime acid, and ceftazidime are the firstand third-generation parenteral cephalosporins. Cefdinir, however, is a third-generation oral cephalosporin having up to $25 \%$ bioavailability. ${ }^{60}$ A number of cephalosporins have been proved to possess remarkable activity against a number of bacteria, including B. burgdorferi. ${ }^{35}$

Another group of agents that were found to be active against the stationary phase of B. burgdorferi, with $>97 \%$ inhibition, were the mobile ion carriers or ionophores. 
Valinomycin and calcimycin act as ionophores that mediate the transport of charged molecules across the bacterial cell wall causing an imbalance in the electrochemical gradient. ${ }^{61}$ Gramicidin, following a similar mechanism of action, tends to form a channel in the bacterial wall and help in the transfer of ions across the bacterial membrane. All three drug molecules, valinomycin, calcimycin, and gramicidin, along with osmotic imbalance cause an associated disruption in the normal oxidative phosphorylation process of the bacterial cell leading, ultimately, to the death of the bacteria. Though the toxicity of ionophores is generally very well known in living systems, these drug molecules can be investigated for their potential to be used in combination therapy against various serious infectious diseases.

An interesting drug candidate identified in the study was the alcohol dehydrogenase inhibitor, disulfiram. Though the drug is used as a treatment of alcohol abuse, recently its anticancer potential has also been discovered. It has been known to make complexes with metal ions and cause the disruption of the proteasome, leading to death of the cancer cells. Similarly, the metabolites of the drug molecule have been known to significantly inhibit the growth of a number of bacterial species, including the biofilm-forming Pseudomonas aeruginosa. ${ }^{62}$ The drug molecule has a good bioavailability, and it passes the blood-brain barrier to show its effect in the central nervous system. Though most of the studies are concerned to the use of the drug molecule in alcoholism yet, being a safe, FDA-approved molecule, the drug can be repurposed for its antibacterial potential.

As we observe that azlocillin sodium and cefotaxime acid show low MIC values, we studied time kill studies to determine the rate of antimicrobial activity. In our observation, both azlocillin sodium and cefotaxime acid reduced Borrelia no 3- $\log 10$-unit (99.9\%) between 48 hours and 72 hours at concentrations $2.5 \mu \mathrm{M}$ and $5 \mu \mathrm{M}$, respectively.

The identified compounds can potentially provide effective antibacterial cover against the persister forms of B. burgdorferi, if indeed such forms can be proven to exist in patients treated for Lyme disease who remain symptomatic. The drug molecules exploit multiple mechanisms to show their inhibitory effects. Moreover, due to the broad variety of molecules, multiple routes of administration and dosage forms can be developed for effectively treating the Lyme disease infections. In summary, this study has provided a mean to screen a large number of compounds using a highly sensitive, reliable, and rapid platform. The assay has identified a number of FDA-approved drug molecules with their potential to be repurposed for use against both Lyme disease and the associated PTLDS. In addition to identify drugs and evaluate the MIC and MBC values, further studies should be done by using in vitro persisters' model to prove whether these drugs have potential to eliminate persisters or not. ${ }^{21}$ However, additional preclinical and clinical data can substantiate the use of these drug molecules for repurposing and clinical acceptance, thereafter.

\section{Acknowledgments}

This work was accomplished with a generous grant from the Bay Area Lyme Foundation. The authors are indebted to Dr Robert Lane (UC Berkley, Berkley, CA, USA) for his constant support, discussion, and provision of valuable bacterial strains for the study.

\section{Disclosure}

Venkata Raveendra Pothineni and Jayakumar Rajadas are listed on the following disclosure \# S16-018 "New drug combination candidates against B. burgdorferi, B. duttonii, B. garinii, B. afzelii and B. miyamotoi for the treatment of Acute and as Post-Treatment Lyme Disease Syndrome (PTLDS)" under patent application no 62/279,826 assigned to Stanford University. All other authors report no conflicts of interest in this work.

\section{References}

1. Kuehn BM. CDC estimates 300,000 US cases of Lyme disease annually. JAMA. 2013;310:1110.

2. Vollmer SA, Feil EJ, Chu CY, et al. Spatial spread and demographic expansion of Lyme borreliosis spirochaetes in Eurasia. Infect Genet Evol. 2013;14:147-155.

3. Brownstein JS, Holford TR, Fish D. Effect of climate change on Lyme disease risk in North America. Ecohealth. 2005;2:38-46.

4. Esposito S, Bosis S, Sabatini C, Tagliaferri L, Principi N. Borrelia burgdorferi infection and Lyme disease in children. Int J Infect Dis. 2013;17:e153-e158.

5. Borchers AT, Keen CL, Huntley AC, Gershwin ME. Lyme disease: a rigorous review of diagnostic criteria and treatment. J Autoimmun. 2015; 57:82-115.

6. Bockenstedt LK, Radolf JD. Xenodiagnosis for posttreatment Lyme disease syndrome: resolving the conundrum or adding to it? Clin Infect Dis. 2014;58:946-948.

7. Feng J, Wang T, Shi W, et al. Identification of novel activity against Borrelia burgdorferi persisters using an FDA approved drug library. Emerg Microbes Infect. 2014;3:e49.

8. Bockenstedt LK, Gonzalez DG, Haberman AM, Belperron AA. Spirochete antigens persist near cartilage after murine Lyme borreliosis therapy. J Clin Invest. 2012;122:2652-2660.

9. Hodzic E, Feng S, Holden K, Freet KJ, Barthold SW. Persistence of Borrelia burgdorferi following antibiotic treatment in mice. Antimicrob Agents Chemother. 2008;52:1728-1736.

10. Diterich I, Rauter C, Kirschning CJ, Hartung T. Borrelia burgdorferiinduced tolerance as a model of persistence via immunosuppression. Infect Immun. 2003;71:3979-3987.

11. Steere AC, Schoen RT, Taylor E. The clinical evolution of Lyme arthritis. Ann Intern Med. 1987;107:725-731.

12. Barthold SW, de Souza MS, Janotka JL, Smith AL, Persing DH. Chronic Lyme borreliosis in the laboratory mouse. Am J Pathol. 1993;143: 959-971. 
13. Babady NE, Hall L, Abbenyi AT, et al. Evaluation of Mycobacterium avium complex clarithromycin susceptibility testing using SLOMYCO Sensititre panels and JustOne strips. J Clin Microbiol. 2010;48: $1749-1752$.

14. Embers ME, Barthold SW, Borda JT, et al. Persistence of Borrelia burgdorferi in rhesus macaques following antibiotic treatment of disseminated infection. PLoS One. 2012;7:e29914.

15. Hodzic E, Imai D, Feng S, Barthold SW. Resurgence of persisting noncultivable Borrelia burgdorferi following antibiotic treatment in mice. PLoS One. 2014;9:e86907.

16. Straubinger RK, Summers BA, Chang YF, Appel MJ. Persistence of Borrelia burgdorferi in experimentally infected dogs after antibiotic treatment. J Clin Microbiol. 1997;35:111-116.

17. Marques A, Telford SR 3rd, Turk SP, et al. Xenodiagnosis to detect Borrelia burgdorferi infection: a first-in-human study. Clin Infect Dis. 2014;58:937-945.

18. Brorson Ø, Brorson SH, Scythes J, MacAllister J, Wier A, Margulis L. Destruction of spirochete Borrelia burgdorferi round-body propagules (RBs) by the antibiotic tigecycline. Proc Natl Acad Sci U S A. 2009;106: 18656-18661.

19. Miklossy J, Kasas S, Zurn AD, McCall S, Yu S, McGeer PL. Persisting atypical and cystic forms of Borrelia burgdorferi and local inflammation in Lyme neuroborreliosis. J Neuroinflammation. 2008;5:1-18.

20. Lantos PM, Auwaerter PG, Wormser GP. A systematic review of Borrelia burgdorferi morphologic variants does not support a role in chronic Lyme disease. Clin Infect Dis. 2014;58:663-671.

21. Sharma B, Brown AV, Matluck NE, Hu LT, Lewis K. Borrelia burgdorferi, the causative agent of Lyme disease, forms drug-tolerant persister cells. Antimicrob Agents Chemother. 2015;59:4616-4624.

22. Feng J, Auwaerter PG, Zhang Y. Drug combinations against Borrelia burgdorferi persisters in vitro: eradication achieved by using daptomycin, cefoperazone and doxycycline. PLoS One. 2015;10:e0117207.

23. Sapi E, Kaur N, Anyanwu S, et al. Evaluation of in-vitro antibiotic susceptibility of different morphological forms of Borrelia burgdorferi. Infect Drug Resist. 2011;4:97-113.

24. Pavia C, Inchiosa MA Jr, Wormser GP. Efficacy of short-course ceftriaxone therapy for Borrelia burgdorferi infection in $\mathrm{C} 3 \mathrm{H}$ mice. Antimicrob Agents Chemother. 2002;46:132-134.

25. Wormser GP, Daniels TJ, Bittker S, Cooper D, Wang G, Pavia CS. Failure of topical antibiotics to prevent disseminated Borrelia burgdorferi infection following a tick bite in C3H/HeJ mice. J Infect Dis. 2012;205:991-994.

26. Pavia CS, Wormser GP. Culture of the entire mouse to determine whether cultivable Borrelia burgdorferi persists in infected mice treated with a five-day course of Ceftriaxone. Antimicrob Agents Chemother. 2014;58:6701-6703.

27. Lefas G, Chaconas G. High-throughput screening identifies three inhibitor classes of the telomere resolvase from the Lyme disease spirochete. Antimicrob Agents Chemother. 2009;53:4441-4449.

28. Cornell KA, Primus S, Martinez JA, Parveen N. Assessment of methylthioadenosine/S-adenosylhomocysteine nucleosidases of Borrelia burgdorferi as targets for novel antimicrobials using a novel high-throughput method. J Antimicrob Chemother. 2009;63: $1163-1172$.

29. Feng J, Shi W, Zhang S, Zhang Y. Identification of new compounds with high activity against stationary phase Borrelia burgdorferi from the NCI compound collection. Emerg Microbes Infect. 2015;4:e31.

30. Feng J, Weitner M, Shi W, Zhang S, Sullivan D, Zhang Y. Identification of additional anti-persister activity against Borrelia burgdorferi from an FDA drug library. Antibiotics. 2015;4:397.

31. Nygren P, Larsson R. Drug repositioning from bench to bedside: tumour remission by the antihelmintic drug mebendazole in refractory metastatic colon cancer. Acta Oncol. 2014;53:427-428.

32. Jahchan NS, Dudley JT, Mazur PK, et al. A drug repositioning approach identifies tricyclic antidepressants as inhibitors of small cell lung cancer and other neuroendocrine tumors. Cancer Discov. 2013;3: 1364-1377.
33. Wagh D, Pothineni VR, Inayathullah M, Liu S, Kim KM, Rajadas J. Borreliacidal activity of Borrelia metal transporter A (BmtA) binding small molecules by manganese transport inhibition. Drug Des Devel Ther. 2015;9:805.

34. Kraiczy P, Weigand J, Wichelhaus TA, et al. In vitro activities of fluoroquinolones against the spirochete Borrelia burgdorferi. Antimicrob Agents Chemother. 2001;45:2486-2494.

35. Hunfeld KP, Rodel R, Wichelhaus TA. In vitro activity of eight oral cephalosporins against Borrelia burgdorferi. Int J Antimicrob Agents. 2003;21:313-318.

36. Veinovic G, Cerar T, Strle F, et al. In vitro susceptibility of European human Borrelia burgdorferi sensu stricto strains to antimicrobial agents. Int J Antimicrob Agents. 2013;41:288-291.

37. Feng J, Wang T, Zhang S, Shi W, Zhang Y. An optimized SYBR Green I/PI assay for rapid viability assessment and antibiotic susceptibility testing for Borrelia burgdorferi. PLoS One. 2014;9:e111809.

38. Feng J, Wang T, Shi W, et al. Identification of novel activity against Borrelia burgdorferi persisters using an FDA approved drug library. Emerg Microbes Infect. 2014;3:e49.

39. Marques A. Chronic Lyme disease: a review. Infect Dis Clin North Am. 2008;22:341-360,vii-viii.

40. Terekhova D, Sartakova ML, Wormser GP, Schwartz I, Cabello FC. Erythromycin resistance in Borrelia burgdorferi. Antimicrob Agents Chemother. 2002;46:3637-3640.

41. Kannan K, Kanabar P, Schryer D, et al. The general mode of translation inhibition by macrolide antibiotics. Proc Natl Acad Sci U S A. 2014; 111:15958-15963.

42. Steel HC, Theron AJ, Cockeran R, Anderson R, Feldman C. Pathogenand host-directed anti-inflammatory activities of macrolide antibiotics. Mediators Inflamm. 2012;2012:584262.

43. Shinkai M, Henke MO, Rubin BK. Macrolide antibiotics as immunomodulatory medications: proposed mechanisms of action. Pharmacol Ther. 2008;117:393-405.

44. Leach KL, Brickner SJ, Noe MC, Miller PF. Linezolid, the first oxazolidinone antibacterial agent. Ann N Y Acad Sci. 2011;1222:49-54.

45. Van Bambeke F. Macrolides and ketolides. In: Vinks A, Derendorf H, Mouton JW, editors. Fundamentals of Antimicrobial Pharmacokinetics and Pharmacodynamics. New York, NY: Springer; 2014:257-278.

46. Yuan-shu Q, Qi-nan W, Yu-fu J. Pharmacokinetic study of leucomycin in healthy volunteers [J]. Zhongguo Kang Sheng Su Za Zhi. 1990;2:008.

47. Lin AH, Murray RW, Vidmar TJ, Marotti KR. The oxazolidinone eperezolid binds to the $50 \mathrm{~S}$ ribosomal subunit and competes with binding of chloramphenicol and lincomycin. Antimicrob Agents Chemother. 1997; 41:2127-2131.

48. Dryden MS. Linezolid pharmacokinetics and pharmacodynamics in clinical treatment. J Antimicrob Chemother. 2011;66(Suppl 4):iv7-iv15.

49. Keel RA, Schaeftlein A, Kloft C, et al. Pharmacokinetics of intravenous and oral linezolid in adults with cystic fibrosis. Antimicrob Agents Chemother. 2011;55:3393-3398.

50. Viaggi B, Paolo AD, Danesi R, et al. Linezolid in the central nervous system: comparison between cerebrospinal fluid and plasma pharmacokinetics. Scand J Infect Dis. 2011;43:721-727.

51. Kutscha-Lissberg F, Hebler U, Muhr G, Köller M. Linezolid penetration into bone and joint tissues infected with methicillin-resistant staphylococci. Antimicrob Agents Chemother. 2003;47:3964-3966.

52. Pérez-Arnaiz C, Busto N, Leal JM, García B. New insights into the mechanism of the DNA/doxorubicin interaction. J Phys Chem B. 2014; 118:1288-1295.

53. Kizek R, Adam V, Hrabeta J, et al. Anthracyclines and ellipticines as DNA-damaging anticancer drugs: recent advances. Pharmacol Ther. 2012;133:26-39.

54. Danesi R, Fogli S, Gennari A, Conte P, Del Tacca M. Pharmacokineticpharmacodynamic relationships of the anthracycline anticancer drugs. Clin Pharmacokinet. 2002;41:431-444.

55. Pucci MJ, Wiles JA. Bacterial Topoisomerase Inhibitors: Quinolones and Beyond. Antimicrobials. New York, NY: Springer; 2014:307-326. 
56. Stass H, Kubitza D. Pharmacokinetics and elimination of moxifloxacin after oral and intravenous administration in man. J Antimicrob Chemother. 1999;43(Suppl B):83-90.

57. Malik M, Drlica K. Moxifloxacin lethality against Mycobacterium tuberculosis in the presence and absence of chloramphenicol. Antimicrob Agents Chemother. 2006;50:2842-2844.

58. Niki Y. Pharmacokinetics and safety assessment of tosufloxacin tosilate. J Infect Chemother. 2002;8:1-18.

59. Fernandes R, Amador P, Prudêncio C. $\beta$-Lactams: chemical structure, mode of action and mechanisms of resistance. Rev Med Microbiol. 2013; 24:7-17.
60. Guay DR. Cefdinir: an expanded-spectrum oral cephalosporin. Ann Pharmacother. 2000;34:1469-1477.

61. Berezin SK. Valinomycin as a classical anionophore: mechanism and ion selectivity. J Membr Biol. 2015;248:713-726.

62. Zaldívar-Machorro VJ, López-Ortiz M, Demare P, Regla I, Muñoz-Clares RA. The disulfiram metabolites S-methyl-N,Ndiethyldithiocarbamoyl sulfoxide and S-methyl-N,N-diethylthiocarbamoyl sulfone irreversibly inactivate betaine aldehyde dehydrogenase from Pseudomonas aeruginosa, both in vitro and in situ, and arrest bacterial growth. Biochimie. 2011;93:286-295. 


\section{Supplementary material}

Table SI List of identified compounds showing $>95 \%$ inhibition

\begin{tabular}{|c|c|c|c|}
\hline Serial number & $\%$ of inhibition & Compound name & Library source \\
\hline I & 95.3 & Ceftriaxone sodium & LOPAC \\
\hline 2 & 90.53 & Diphenyleneiodonium chloride & LOPAC \\
\hline 3 & 94.14 & Doxycycline hydrochloride & LOPAC \\
\hline 4 & 95.91 & Idarubicin & LOPAC \\
\hline 5 & 98.59 & Mitoxantrone & LOPAC \\
\hline 6 & 97.12 & Minocycline hydrochloride & LOPAC \\
\hline 7 & 97.19 & PD 404, I82 & LOPAC \\
\hline 8 & 98.37 & Ammonium pyrrolidinedithiocarbamate & LOPAC \\
\hline 9 & 98.55 & Vancomycin hydrochloride & LOPAC \\
\hline 10 & 96.74 & WB 64 & LOPAC \\
\hline 11 & 98.16 & Erythromycin & Microsource \\
\hline 12 & 96.78 & Actinomycin D & Microsource \\
\hline 13 & 98.52 & Mitomycin C & Microsource \\
\hline 14 & 97 & Nafcillin sodium & Microsource \\
\hline 15 & 96.62 & Dirithromycin & Microsource \\
\hline 16 & 91.11 & Norfloxacin & Microsource \\
\hline 17 & 99.1 & Cefoxitin sodium & Microsource \\
\hline 18 & 96.94 & Amoxicillin & Microsource \\
\hline 19 & 95.44 & Clarithromycin & Microsource \\
\hline 20 & 93.93 & Ampicillin sodium & Microsource \\
\hline 21 & 96.47 & Novobiocin sodium & Microsource \\
\hline 22 & 93.67 & Oxacillin sodium & Microsource \\
\hline 23 & 98.09 & Oxytetracycline & Microsource \\
\hline 24 & 97.34 & Meclocycline sulfosalicylate & Microsource \\
\hline 25 & 97.52 & Gemifloxacin mesylate & Microsource \\
\hline 26 & 96.89 & Methacycline hydrochloride & Microsource \\
\hline 27 & 98.03 & Cephapirin sodium & Microsource \\
\hline 28 & 95.24 & Puromycin hydrochloride & Microsource \\
\hline 29 & 99.63 & Josamycin & Microsource \\
\hline 30 & 95.2 & Chloramphenicol palmitate & Microsource \\
\hline 31 & 96.3 & Chloramphenicol hemisuccinate & Microsource \\
\hline 32 & 98.27 & Chloramphenicol & Microsource \\
\hline 33 & 98.79 & Chlorhexidine & Microsource \\
\hline 34 & 96.55 & Benzoic acid, 2-hydroxy compound with 8-quinolinol (I:I) & Microsource \\
\hline 35 & 97.71 & Penicillin G potassium & Microsource \\
\hline 36 & 98.23 & Penicillin V potassium & Microsource \\
\hline 37 & 98.64 & Piperacillin sodium & Microsource \\
\hline 38 & 97.98 & Clindamycin hydrochloride & Microsource \\
\hline 39 & 98.39 & Cloxacillin sodium & Microsource \\
\hline 40 & 98.71 & Tyrothricin & Microsource \\
\hline $4 I$ & 98.84 & Vancomycin hydrochloride & Microsource \\
\hline 42 & 97.97 & Phenylmercuric acetate & Microsource \\
\hline 43 & 99.67 & Phenethicillin potassium & Microsource \\
\hline 44 & 97.24 & Hetacillin potassium & Microsource \\
\hline 45 & 97.1 & Daunorubicin & Microsource \\
\hline 46 & 97.78 & Demeclocycline hydrochloride & Microsource \\
\hline 47 & 98.8 & Oleandomycin phosphate & Microsource \\
\hline 48 & 96.61 & Dicloxacillin sodium & Microsource \\
\hline 49 & 98.02 & Spectinomycin U & Microsource \\
\hline 50 & 97.95 & Aminacrine & Microsource \\
\hline 51 & 98.73 & Pyrithione zinc & Microsource \\
\hline 52 & 97.49 & Lincomycin hydrochloride & Microsource \\
\hline 53 & 99.19 & Tetracycline hydrochloride & Microsource \\
\hline 54 & 97.88 & Methicillin sodium & Microsource \\
\hline 55 & 93.86 & Thioguanine & Microsource \\
\hline
\end{tabular}

(Continued) 
Table SI (Continued)

\begin{tabular}{|c|c|c|c|}
\hline Serial number & $\%$ of inhibition & Compound name & Library source \\
\hline 56 & 92.47 & Hycanthone mesylate & Microsource \\
\hline 57 & 98.37 & Erythromycin estolate & Microsource \\
\hline 58 & 96.1 & Gatifloxacin & Microsource \\
\hline 59 & 98.11 & Roxithromycin & Microsource \\
\hline 60 & 98.18 & Mitoxantrone & Microsource \\
\hline 61 & 97.68 & Moxifloxacin hydrochloride & Microsource \\
\hline 62 & 98.45 & Tilmicosin & Microsource \\
\hline 63 & 98.14 & Monensin sodium (monensin $\mathrm{A}$ is shown) & Microsource \\
\hline 64 & 97.89 & Spiramycin & Microsource \\
\hline 65 & 98.59 & Azithromycin & Microsource \\
\hline 66 & 98.37 & Ramipril & Microsource \\
\hline 67 & 96.78 & Bacampicillin hydrochloride & Microsource \\
\hline 68 & 98.21 & Ceftriaxone sodium trihydrate & Microsource \\
\hline 69 & 98.16 & Telithromycin & Microsource \\
\hline 70 & 97.76 & Oxaprozin & Microsource \\
\hline 71 & 97.81 & Thiamphenicol & Microsource \\
\hline 72 & 97.8 & Tylosin tartrate & Microsource \\
\hline 73 & 92.47 & Sarafloxacin hydrochloride & Microsource \\
\hline 74 & 98.37 & Cefditorin pivoxil & Microsource \\
\hline 75 & 98.86 & Montelukast sodium & Microsource \\
\hline 76 & 88.63 & Khayanthone & Microsource \\
\hline 77 & 98.23 & Ceftazidime & Microsource \\
\hline 78 & 98.56 & Pyrromycin I-naphthacenecarboxylic acid & Microsource \\
\hline 79 & 98.3 & Khivorin & Microsource \\
\hline 80 & 95.3 & Kitasamycin & NIHCC \\
\hline 81 & 97.85 & Florfenicol & Microsource \\
\hline 82 & 98.19 & Actinonin & Microsource \\
\hline 83 & 76.96 & Enrofloxacin & Microsource \\
\hline 84 & 99.2 & Cefazolin sodium & Microsource \\
\hline 85 & 98.61 & Alexidine hydrochloride & Microsource \\
\hline 86 & 93.81 & Pristimerin & Microsource \\
\hline 87 & 97.69 & Salinomycin, sodium & Microsource \\
\hline 88 & 98.69 & Dromilac ethidium bromide & Microsource \\
\hline 89 & 99.05 & Cephalosporin c sodium & Microsource \\
\hline 90 & 97.6 & Erythromycin stearate & Microsource \\
\hline 91 & 97.09 & Agelasine (stereochemistry of diterpene unknown) & Microsource \\
\hline 92 & 99.5 & Diphenyleneiodonium chloride & Biomol \\
\hline 93 & 98.24 & Actinomycin D & Biomol \\
\hline 94 & 96.28 & Puromycin & Biomol \\
\hline 95 & 93.86 & Hoechst 33342 (cell permeable) (bisbenzimide) & Biomol \\
\hline 96 & 92.74 & Gliotoxin & Biomol \\
\hline 97 & 97 & Mitomycin C & Biomol \\
\hline 98 & 96.68 & Monensin & Biomol \\
\hline 99 & 99.55 & Clindamycin hydrochloride & Biomol \\
\hline 100 & 98.29 & Lincomycin & Biomol \\
\hline 101 & 96.85 & Novobiocin sodium & Biomol \\
\hline 102 & 98.51 & Troleandomycin & Biomol \\
\hline 103 & 96.51 & Aclacinomycin & Biomol \\
\hline 104 & 98.17 & Spectinomycin & Biomol \\
\hline 105 & 98.43 & A-237I & Biomol \\
\hline 106 & 96.01 & Mitomycin C & Biomol \\
\hline 107 & 98.37 & Puromycin & Biomol \\
\hline 108 & 98.25 & Daunorubicin hydrochloride & Biomol \\
\hline 109 & 96.63 & Bortezomib & Biomol \\
\hline 110 & 98.96 & Auranofin & Biomol \\
\hline III & 98.96 & Meropenem & Biomol \\
\hline 112 & 99.72 & Mitoxantrone & Biomol \\
\hline 113 & 99.34 & Tylosin tartrate & Biomol \\
\hline
\end{tabular}


Table SI (Continued)

\begin{tabular}{|c|c|c|c|}
\hline Serial number & $\%$ of inhibition & Compound name & Library source \\
\hline 114 & 96.94 & Thiamphenicol glycinate & Biomol \\
\hline 115 & 98.15 & Oxacillin sodium & Biomol \\
\hline 116 & 97.22 & Penicillin V potassium & Biomol \\
\hline 117 & 98.73 & Piperacillin & Biomol \\
\hline 118 & 99.47 & Florfenicol & Biomol \\
\hline 119 & 98.48 & Ampicillin & Biomol \\
\hline 120 & 98.64 & Azithromycin & Biomol \\
\hline $12 \mid$ & 96.99 & Gatifloxacin & Biomol \\
\hline 122 & 97.5 & Ceftazidime & Biomol \\
\hline 123 & 96.94 & Chloramphenicol & Biomol \\
\hline 124 & 97.77 & Roxithromycin & Biomol \\
\hline 125 & 97.6 & Sparfloxacin & Biomol \\
\hline 126 & 95.68 & Ciprofloxacin & Biomol \\
\hline 127 & 98.79 & Clarithromycin & Biomol \\
\hline 128 & 97.14 & Clinafloxacin & Biomol \\
\hline
\end{tabular}

Abbreviations: LOPAC, Library of Pharmacologically Active Compounds; NIHCC, NIH Clinical Collection.

\section{Publish your work in this journal}

Drug Design, Development and Therapy is an international, peerreviewed open-access journal that spans the spectrum of drug design and development through to clinical applications. Clinical outcomes, patient safety, and programs for the development and effective, safe, and sustained use of medicines are a feature of the journal, which has also been accepted for indexing on PubMed Central. The manuscript management system is completely online and includes a very quick and fair peer-review system, which is all easy to use. Visit http://www.dovepress.com/testimonials.php to read real quotes from published authors.

Submit your manuscript here: http://www.dovepress.com/drug-design-development-and-therapy-journal 